\section{A EXPERIÊNCIA DA PESQUISA ENSINASUS}

THE EXPERIENCE OF ENSINASUS RESEARCH

\author{
Lilian Koifman 1 \\ Regina Lúcia Monteiro Henriques 2
}

Resumo Este artigo descreve a trajetória do projeto EnsinaSUS, da pesquisa desenvolvida em seu interior e das múltiplas dimensões e implicações na direção do conhecimento das experiências que têm sido realizadas nos cursos da área de saúde em relação aos processos de mudança que estão em desenvolvimento. Pretendemos compartilhar a experiência de integração de pesquisadores, de diferentes origens institucionais e profissionais, na construção de um projeto comum, alimentado da vivência desses atores. Essa experiência almejou inovar e construir uma nova gramática para discussão e compreensão dos processos pedagógicos e sua interface com a saúde e o trabalho, na produção de um cuidado que se faça distinto na concepção da assistência à saúde, produzindo cuidado usuáriocentrado, numa perspectiva dialógica e ético-política. Entendemos que as práticas de ensino e extensão funcionam como dispositivos abertos de um 'fazer-pensar-saber' em integralidade, forjando novas concepções, sentidos e significados da saúde, da vida, do conhecimento e da educação. Pautamonos na idéia de que as explicações da práxis pedagógica podem ser entendidas como possibilidade da construção histórica da cidadania. Encontramos alguns mecanismos de legitimação e canais de articulação, intercâmbio e solidariedade como resposta de interação de diversidades. A equipe representou a composição de redes de representação em espaços culturais e políticos plurais.

Palavras-chave formação em saúde; metodologia de pesquisa em saúde; integralidade.
Abstract This article describes the EnsinasuS Project, the research developed within the project, and the various dimensions and implications that will lead to understanding the experiences that have been taking place in courses in the field of health care as regards their processes of change. Our aim is to share the experience of integrating researchers, coming from different institutional and professional backgrounds, in order to build a common project, based on their experience. The purpose of this project was to innovate and to build a new grammar so as to discuss and understand pedagogical processes and their interface with health and work, and so produce health care which is different in its concept of health assistance, thus producing a user-centred care, from a dialogical and politically-ethical viewpoint. We believe that teaching and extension practices open up an 'action-thought-knowledge' overall concept, promoting new concepts and meanings for health, life, knowledge and education. We also believe that explanations from pedagogical praxis may be seen as a possibility of historically building citizenship. We found that some mechanisms of legitimation and modes of articulation, exchange and solidarity are key to the interaction of diversity. The team was composed of representation networks from different political and cultural environments.

Keywords formation for health care workers; health research methodology; integrality. 


\section{Antecedentes}

O EnsinaSUS - ensino, desenvolvimento, pesquisa e documentação na construção da integralidade da atenção à saúde - é um dos projetos desenvolvidos pelo Laboratório de Pesquisas sobre Práticas de Integralidade em Saúde (Lappis), do Instituto de Medicina Social (IMS) da Universidade Estadual do Estado do Rio de Janeiro (Uerj). Institucionalizado em 2003, e integrando o grupo de pesquisadores Racionalidades Médicas, do Conselho Nacional de Desenvolvimento Científico e Tecnológico (CNPq), o laboratório visa a agregar grupos de pesquisadores interessados nos estudos sobre a integralidade em uma de suas dimensões, assim como estreitar laços (inter)institucionais, a partir da construção de projetos integrados, como componentes de suas linhas de atuação, entre as quais está a linha da Formação. Cabe frisar que o projeto Integralidade e o Lappis contam, desde a sua origem, com o apoio do CNPq e da Fundação Carlos Chagas Filho de Amparo à Pesquisa do Estado do Rio de Janeiro (Faperj), além do Ministério da Saúde (MS) e da Uerj.

Daremos destaque à descrição da linha Formação, pois é nela que se insere a pesquisa descrita no presente artigo. O EnsinaSUS, assim, é um dos 'projetos-movimento' que buscou mapear e sistematizar estudos sobre currículos profissionais voltados para a atenção integral à saúde individual e coletiva e integralidade do cuidado em saúde. Teve também como objetivo mapear os efeitos de produção de subjetividade em suas diferentes inserções, no que concerne ao estabelecimento de apoio às experiências inovadoras no campo da formação e da educação permanente em saúde. A partir do mapeamento e da sistematização dos estudos, almejou desenvolver metodologias para a realização de análises dessas e de outras experiências capazes de subsidiar as transformações nos processos de educação dos profissionais de saúde.

Referimo-nos a 'projeto-movimento', pois a produção de conhecimento resultante dos saberes e das práticas do ensino da saúde, para a integralidade, requer uma construção teórica contextualizada em movimentos de vontade capazes de forjar uma teoria viva que reflita, desde a sua própria constituição, a experiência concreta da vida que lhe dá origem e da vida que vai originar, uma atividade prática (práxis), uma iniciativa da vontade, um movimento de transformação (Pinheiro e Ceccim, 2006).

$\mathrm{Na}$ análise sistemática do nosso objeto de pesquisa, foi possível localizar coerências com as proposições teóricas e entre as proposições observacionais, umas com as outras nas próprias experiências, além de revelar correspondência com as realidades experienciadas pelos pesquisadores e por outros coletivos com interrogações semelhantes. Possibilitou novas 
leituras sobre o cotidiano para atores implicados em práticas de ensino da integralidade em saúde (Pinheiro et al., 2006).

O EnsinaSUS originou-se de uma convergência do movimento do próprio grupo de pesquisa do Lappis e do MS, através de uma política do Sistema Único de Saúde (SUS) para o diálogo com o ensino de graduação nas profissões da área de saúde: AprenderSUS (Pinheiro et al., 2006). De um lado, o Lappis, destacava a importância da formação profissional para a concretização do princípio da integralidade, a partir dos estudos sobre experiências inovadoras. De outro, o MS, na tentativa de cumprir o mandamento constitucional segundo o qual cabe ao SUS ordenar a formação de recursos humanos para a saúde, buscava formular uma política voltada para a mudança do ensino das profissões de saúde, centrada no princípio da integralidade. Nessa ocasião, buscou-se uma sinergia de esforços, sobretudo no que diz respeito à análise de experiências inovadoras no ensino pautadas pela integralidade.

Após vários encontros, a equipe de pesquisadores do Lappis estruturou o projeto EnsinaSUS, iniciado em janeiro de 2004.

Este projeto teve por objetivo geral apoiar experiências inovadoras, realizadas por instituições de ensino e pesquisa, para a melhoria da educação dos profissionais de saúde em dois campos de atuação específicos: formação e educação permanente dos profissionais, tendo em vista as interfaces educação, saúde e trabalho; e desenvolvimento e incorporação de novas tecnologias do cuidado, capazes de articular saberes e práticas, produzidos nos serviços como estratégias para conhecê-las, avaliá-las e promover sua divulgação.

Para melhor operacionalização do projeto, organizou-se um conjunto de estratégias com o agrupamento das seguintes atividades:

- Análise das experiências inovadoras no campo da formação e da educação permanente em saúde, mediante a organização de convocatória para apresentação de propostas curriculares e metodológicas, no ensino de graduação, pelas instituições de ensino superior (IES), e no ensino técnico, pelas escolas técnicas de saúde (ETS), que enfatizem a integralidade em saúde.

- Desenvolvimento de metodologias e capacitações e de subsídios à construção e avaliação de currículos integrados em saúde.

- Disseminação e divulgação das experiências inovadoras de integralidade na interface educação-saúde-trabalho, assim como estabelecimento de um fórum permanente de discussão crítica e reflexiva sobre a formação e desenvolvimento profissional. 
No intuito de permitir melhor incremento do Projeto de Pesquisa EnsinaSUS, foram organizadas as tarefas da equipe de pesquisadores. Dela fizeram parte: pesquisadores das instituições envolvidas com a linha de atuação EnsinaSUS - Instituto de Medicina Social e Faculdade de Enfermagem, ambos da Uerj, Laboratório Universitário de Planejamento em Saúde (Lupa) do Departamento de Planejamento em Saúde do Instituto de Saúde da Comunidade da Universidade Federal Fluminense (UFF) e Grupo Temático de Saúde da Faculdade de Educação da Universidade Federal do Rio Grande do Sul (UFRGS). Além dos participantes acima listados, a equipe teve reuniões nas quais se somaram outros professores, residentes e estudantes de graduação das instituições envolvidas.

Os produtos e resultados parciais gerados subdividem-se em três ações específicas, mas não excludentes entre si: pesquisa empírica; desenvolvimento de metodologias e capacitações pedagógicas; e produção e disseminação de informações (bibliográfica, científica, documental e institucional) para divulgação das discussões desenvolvidas nessa área. Desse modo, podemos considerar que a meta primordial desse projeto consiste em inaugurar um dispositivo germinador de estudos voltados para as práticas de integralidade no SUS, sendo o campo da formação capaz de produzir conhecimento, novos saberes e subjetivação profissional, coletiva e institucional.

Para conhecer as trajetórias e a materialidade dessas experiências, assim como identificar os atores-autores e suas práticas nos processos de educação dos profissionais de saúde, optou-se por uma abordagem transdisciplinar, baseada principalmente nas ciências humanas e sociais em saúde. Buscou-se traçar um mapa das ações e dos atores envolvidos (estudantes, profissionais, docentes e usuários), a partir de uma análise dos processos realizados na formação profissional, dos desenhos pedagógicos utilizados, das interfaces estabelecidas entre educação, saúde e trabalho e as respectivas inscrições nos campos educacional, sanitário, científico, político, social, cultural e econômico.

Dessa forma, entendeu-se ser possível apreender os elementos constituintes das transformações ocorridas no ensino-aprendizagem e na produção de conhecimentos, tão necessários à integralidade em saúde.

No âmbito dos objetivos desta proposta estão o conhecimento e a análise de práticas educacionais e pedagógicas na formação profissional que permitam identificar e possibilitar transformações nos cursos de graduação de diferentes carreiras profissionais.

Como principal referencial, frente à expressão da pluralidade dos atores envolvidos - dirigentes, profissionais, trabalhadores, estudantes e usuários das ações e dos serviços -, destacam-se os usuários como núcleo central das 
ações. Tal centralidade se apóia na integralidade da atenção à saúde, no trabalho em equipe e no conhecimento do sistema de saúde vigente.

A integralidade como conceito estruturante e constituinte de ações que devem orientar as práticas na produção do cuidado, sobretudo no desenvolvimento do trabalho e da educação na saúde, tem em vista um sistema de saúde usuário-centrado. Para tal, torna-se necessário propiciar espaços de produção pedagógica que contribuam para o surgimento de novos sentidos e significados ao agir em saúde.

A característica de circularidade nas e entre as instituições, as teorias e as práticas qualifica a 'roda' das inovações e das mudanças, os elementos e os vetores de suas transformações, seja na integração da rede de serviços, no que concerne ao acolhimento e responsabilização, vínculo, interinstitucionalidade e resolutividade, seja na integração de saberes e práticas que incluem a ampliação das práticas clínicas, o conhecimento da realidade, o trabalho multiprofissional e transdisciplinar, a permeabilidade aos usuários, a ação intersetorial e as práticas locais de ensino e saúde. Nesse sentido, a avaliação das práticas de ensino-aprendizagem, com a adoção de desenhos pedagógicos que privilegiem as interfaces entre educação, saúde e trabalho, constitui marcadores de alta potência capazes de fomentar práticas solidárias de produção de cuidado como ação de cidadania e consolidação do direito à saúde.

A necessidade de mudanças no processo de formação dos trabalhadores da área de saúde se configura como um dos grandes desafios para a conquista de serviços de saúde equânimes, democráticos e acolhedores das necessidades manifestas pelos cidadãos. Nesse sentido, foram desenvolvidos vários movimentos para a produção de transformações substantivas nesse processo, promovidos por associações científicas profissionais, instituições acadêmicas, pelo movimento estudantil e por outros setores da sociedade que se encontram em diferentes estágios nos vários cursos das diferentes profissões da saúde. Nas propostas de formação nessa área, o esforço de superação do antigo modelo biomédico, disciplinar e excessivamente técnico tem sido uma marca importante desse movimento.

Na busca de uma prática voltada para o estabelecimento de uma relação democrática, dialógica e ética no cuidado de saúde, a integralidade e a construção de práticas mais amistosas para promovê-la parecem ser um eixo orientador. Desta forma, as mudanças que se operam nos cursos visitados durante a pesquisa precisam ser entendidas a partir de vários espaços e categorias, com importância e centralidade na construção da possibilidade transformadora. Frente a essa exigência, apresentada pela complexidade dos fenômenos em análise, foram eleitos como eixos prioritários de investigação: a rede de gestão e de serviços com controle social e os saberes e práticas usuário-centradas. 
Propôs-se, com esses eixos, agregar as questões teórico-conceituais e práticas desta pesquisa que foram consideradas relevantes para a formulação de estratégias e a transformação dos processos de ensino-aprendizagem.

Diante dessa compreensão, a rede de serviços e os saberes e práticas que são produzidos e reproduzidos no cotidiano das instituições de saúde podem ser constituídos como espaços de construção da integralidade, na medida em que a integração (presente no interior das concepções de integralidade) seja tomada como idéia-força.

Para orientar a discussão e coleta de dados da pesquisa, foram definidas as seguintes macrocategorias de análise: a relação entre poder, saberes disciplinares e autonomia; o cotidiano e as práticas e saberes; o cuidado; o conhecimento em saúde; e a avaliação ensino-aprendizagem.

As macrocategorias foram utilizadas como marcadores, possibilitando a articulação de conceitos, percepções e sensações para pensar, a partir da pesquisa, outras leituras compreensivas, as quais estão descritas em Pinheiro et al. (2006).

Essas macrocategorias eram estudadas e aprofundadas com a realização de reuniões técnicas, de oficinas de capacitação metodológica com a equipe de pesquisa, contando com a participação de especialistas convidados, além da participação dos pesquisadores em eventos e debates científicos.

Portanto, como dissemos anteriormente, a pesquisa incluiu em seus resultados a análise e a divulgação das experiências selecionadas, com o intuito de compartilhar o conhecimento que tenha potencial para auxiliar a construção de novas práticas locais de ensino pautadas pela integralidade. Isso foi realizado mediante uma reflexão crítica sobre a noção de integralidade, na perspectiva de evitar qualquer noção essencialista do termo. Assumimos a integralidade nas práticas de ensino da saúde como pensamento em ação, um processo de construção que envolve, em si mesmo, o outro e os entornos, a produção e a transformação de saberes e práticas em campo de forças históricas.

Nas várias etapas da pesquisa eram articulados conceitos, percepções e sensações para produzir saberes sobre práticas de ensino orientadas pela integralidade, reconhecendo-se que esse movimento nos requereu a tomada de posição crítica e criativa de identificar possibilidades e desafios (Pinheiro e Ceccim, 2006).

\section{Trajetória}

A pesquisa teve como fio condutor as atividades da linha de atuação do EnsinaSUS do grupo do CNPq-Lappis, iniciada em outubro de 2003, que se 
caracterizou como um projeto com duplo objetivo: mapear e sistematizar estudos sobre currículos profissionais voltados para a integralidade (Pinheiro et al., 2006).

Os representantes dos grupos de pesquisadores do Lappis criaram a linha de atuação voltada para o ensino, o desenvolvimento, a pesquisa e a documentação da construção da integralidade. Como um projeto orgânico, o objetivo geral era mobilizar e apoiar experiências inovadoras, realizadas por instituições de ensino e pesquisa, para a melhoria da educação dos profissionais de saúde, bem como produzir conhecimento relativamente ao ensino em dois campos de atuação específicos: formação e educação permanente dos profissionais, tendo em vista as interfaces entre educação, saúde e trabalho; e desenvolvimento para a incorporação de novas tecnologias do cuidado, capazes de articular saberes e práticas produzidas nos serviços e junto aos usuários.

A dinâmica das atividades propostas no plano de trabalho para atingir tais objetivos foi: a elaboração e o lançamento da convocatória das experiências de práticas de ensino com a realização de uma oficina de divulgação, e a pesquisa científica composta pela pesquisa empírica, por capacitações metodológicas e por atividades de disseminação e divulgação de experiências inovadoras de ensino e de produções científicas sobre o tema.

Na perspectiva apresentada no item anterior sobre as dimensões estruturantes da pesquisa, cabe relatar o seu processo de desenvolvimento no que tange à construção coletiva, marcada pelo intercâmbio entre os pesquisadores e suas experiências particulares, bem como pela aquisição de novos olhares a partir das vivências nas visitas às experiências que participaram da pesquisa.

Ao longo do primeiro ano da pesquisa - 2004 -, iniciaram-se as discussões para a construção e a definição do termo de referência da convocatória das experiências inovadoras de práticas de ensino dos cursos de graduação na área da saúde. As oficinas preparatórias para o lançamento da convocatória e os encontros técnicos foram fundamentais para a delimitação do objeto, a articulação teórico-conceitual e o enfoque metodológico, permitindo a definição do marco lógico e das linhas operatórias de análise. Desse processo surgiu a matriz analítica das experiências. Ainda nesse ano, lançada a convocatória e selecionadas as experiências, construímos coletivamente o trabalho de campo concretizado nos meses de setembro a dezembro de 2004 e de janeiro a abril de 2005.

O segundo ano da pesquisa foi marcado pelo processo de análise, realizado a partir da matriz, com discussões coletivas, oficinas técnicas e de capacitação metodológica para finalização do plano de análise. O encontro com os relatores das experiências participantes da pesquisa ocorreu nesse ano e permitiu a validação da mesma com a utilização da matriz de análise 
para tal fim. O processo de discussão gerou textos analíticos, todos exaustivamente discutidos pelos pesquisadores.

Essas etapas serão detalhadas a seguir.

\section{O campo}

Após a definição do campo em que se daria a pesquisa, teve início o processo de discussão e preparo do termo de referência para a convocatória das experiências inovadoras de práticas de ensino de cursos de graduação da área de saúde. Nesse mesmo período, definiram-se os eixos de análise e campos de atuação nos quais as experiências se situariam, e a partir dos quais poder-se-ia tecer a reflexão sobre as pistas de integralidade presentes nos processos de transformação dos cursos. Os critérios de seleção consideraram quatro requisitos: regionalização, diversidade dos cursos, natureza jurídica e representatividade na força de trabalho em saúde.

O termo da convocatória foi refinado para que pudesse ser utilizado na seleção das experiências. A pesquisa do Lappis foi planejada em conjunto com o lançamento da Política de Formação do MS, com o intuito de potencializar suas ações no sinergismo de idéias para a consecução da oficina nacional. Assim, o seminário de pesquisa foi transformado em oficina com a participação ampla dos diversos atores envolvidos: docentes, estudantes, técnicos do MS, associações de ensino e científicas de várias profissões da área, como medicina, enfermagem, odontologia, psicologia, dentre outras, e movimentos sociais, como movimento estudantil e de educação popular. A oficina foi o momento de divulgação da política do MS para a educação permanente dos profissionais da saúde, bem como o de lançamento e discussão da convocatória para participação dos cursos na pesquisa EnsinaSUS.

Percebemos, com o amadurecimento da proposta de pesquisa e seu dimensionamento, a necessidade de melhor conhecer as duas experiências de transformação dos cursos, ponto de partida e origem dos pesquisadores. Para tanto, como primeira atividade, considerada o marco zero da pesquisa, realizou-se uma reunião técnica na qual foram apresentadas: as experiências pedagógicas da reformulação curricular da Faculdade de Enfermagem da Uerj e a participação do Instituto de Saúde da Comunidade na reestruturação do currículo da Medicina da UFF.

Nesse encontro, ao relatar os avanços, os limites e as possibilidades de transformação nos respectivos cursos e sua relação com a idéia da integralidade, nós - os pesquisadores do Lappis envolvidos nesta pesquisa - fomos provocados a revisitar nossas próprias experiências num intenso e profícuo debate, marcado pela ousadia e pela originalidade, enaltecido pelo despoja- 
mento dos integrantes das equipes em sustentar uma discussão crítica e madura sobre o tema. Com isso, buscou-se superar as diferenças históricas dessas duas categorias profissionais - medicina e enfermagem (juntas, a maior força de trabalho em saúde) -, muitas delas fruto dos confrontos e disputas corporativas no campo da formação e da saúde. O produto dessa reunião foi um relatório técnico acerca da discussão e dos referenciais teóricos e pedagógicos das experiências acima citadas.

Dessa forma, através de reuniões técnicas, oficinas de trabalho, estudo e reflexão dos marcos teóricos da pesquisa, construía-se o roteiro metodológico e, concomitantemente, preparava-se a oficina nacional em conjunto com o MS.

O processo em desenvolvimento delineou a construção do marco analítico capaz de produzir linhas metodológicas e estratégias para análise de experiências inovadoras centradas nas práticas de ensino, o que conduziu as equipes à elaboração de uma matriz de análise.

Ao se perceber a necessidade de aprofundar temas teóricos, professores externos à equipe de pesquisa foram convidados a participar de algumas das reuniões técnicas. Um desses momentos destinou-se à discussão dos temas transversais necessários ao desenvolvimento da análise, como transdisciplinaridade e sua articulação com os dados da pesquisa. Além desse tema, foi possível discutir metodologias de avaliação participativa em ações pedagógicas na área da saúde, o que colaborou com a reflexão em curso e para os primeiros exercícios na matriz de análise revisada em outras três reuniões, finalizando com uma versão a ser cotejada com os eixos da experiência das práticas de ensino.

A equipe, a partir de critérios previamente estabelecidos e de acordo com a convocatória e os campos de atuação definidos, selecionou dez experiências de prática de ensino amistosas à integralidade em saúde, contemplando todas as regiões geopolíticas do país, incluindo a Amazônia Legal.

$\mathrm{Na}$ pesquisa de campo foram realizadas, aproximadamente, 120 entrevistas semi-estruturadas com docentes, discentes, representantes de conselhos municipais de saúde, usuários e gestores, tanto do sistema de saúde como das instituições de ensino. Além das entrevistas, os pesquisadores utilizaram como técnicas de pesquisa: a observação direta do cotidiano do ensino em seus múltiplos cenários de aprendizagem; e os grupos focais com usuários, docentes e discentes de diversos períodos da graduação.

Para a sistematização dos dados, os pesquisadores elaboraram o primeiro plano de análise, à luz das categorias e das dimensões da matriz de análise, definidas na proposta teórico-metodológica da pesquisa. As oficinas de trabalho realizadas permitiram também o compartilhamento do processo de pesquisa em campo, levando à reflexão sobre as dificuldades e as facilidades para obtenção dos dados. A partir desse momento, iniciou-se a 
produção do consolidado da pesquisa em etapas que consistiram em alocar os achados pelas dimensões macro, micro e molecular dos eixos de análise da matriz. Os dados eram refinados a partir da matriz de análise com a participação de todos os pesquisadores e seu mútuo influenciar e interagir. As reuniões ampliadas permitiram identificar dificuldades para a análise do material, e percebeu-se a melhor organização dos eixos de análise para aprofundamento da mesma.

A realização dos encontros, no percurso da análise de dados, foi considerada fundamental para o desenvolvimento de metodologias e capacitações, assim como para a posterior disseminação e divulgação dos resultados de uma produção científica de qualidade e inédita no campo da saúde coletiva.

Com o avanço do processo de análise, vários movimentos permitiram o aprofundamento das dimensões de análise e a reconfiguração do relatório. Foram realizadas oficinas de texto, oficinas para discussão dos relatórios parciais e para a construção dos textos referentes a cada eixo de análise e das macrocategorias.

Outra etapa da pesquisa consistiu no encontro com os representantes das dez experiências pesquisadas, onde através de dinâmicas orientadas validamos os resultados da pesquisa.

O período a seguir foi de intenso trabalho para a organização e a finalização das análises e dos textos que permitiriam a divulgação desse rico material produzido com a utilização de ferramentas metodológicas inovadoras.

Chamamos de inovação metodológica todo processo de preparação da equipe de pesquisadores, sua integração, seleção de referencial teórico e elaboração da matriz de análise. Diferente de outras formas convencionais, a pesquisa nos obrigou a construir novas ferramentas e a realizar diferentes exercícios analíticos, desde a releitura de nossas próprias experiências até a aproximação do material que recebemos durante o prazo de recebimento de experiências da convocatória.

Nosso primeiro 'mapa' foi inscrito pelas próprias experiências nas áreas temáticas e campos de atuação propostos pela convocatória.

O lançamento da convocatória em si cumpriu o nosso principal objetivo: mobilizar experiências dos cursos de graduação em saúde, para conhecê-las e analisá-las, e promover sua divulgação.

Com a seleção teórico-conceitual das categorias operatórias e a construção da matriz propriamente dita, selecionamos as dez experiências, realizamos oficinas e materializamos o que nos parecia algo fundamental e original: ensinar a pesquisa e pesquisar ensinando.

Discutidos exaustivamente pelo grupo de pesquisadores até a confecção final e a edição, os textos produzidos a partir da pesquisa geraram as 
coletâneas: Ensinar saúde: a integralidade e o SUS nos cursos de graduação na área de saúde e Ensino-trabalho-cidadania: novas marcas ao ensinar integralidade no SUS.

\section{Considerações finais}

O encontro das instituições pesquisadoras entre si e com o campo produziu uma nova perspectiva das experiências de ensino, com a qual os coordenadores problematizaram elementos centrais ao processo cotidiano de construção da integralidade.

A idéia de realizar uma pesquisa sobre experiências de ensino da integralidade em saúde na formação de profissionais de saúde surgiu das inquietações relativas à produção do conhecimento científico e à formulação de políticas públicas no campo da formação em saúde. Diante desses questionamentos, optou-se por construir uma pesquisa exploratória que permitisse identificar o potencial inovador das experiências selecionadas.

Mesmo que estejamos conscientes de que não poderemos descrever todos os detalhes e nuanças do percurso da pesquisa, este relato buscou compartilhar o passo-a-passo da experiência do movimento de produção do grupo de pesquisa em sua perspectiva histórica. A partir de diferentes vertentes e convergências de olhares e leituras, a pesquisa produziu um projeto alimentado por vivências e pelo objetivo comum de contribuir para a elaboração de uma nova gramática na discussão e compreensão dos processos pedagógicos por sua interface com a integralidade e o trabalho no SUS (Pinheiro et al., 2006).

Após as visitas ao campo, outros desdobramentos surgiram, a partir de parcerias desenvolvidas. Destacamos como resultados frutíferos, que deram ao trabalho da equipe uma possibilidade de registro visual, a produção do documentário Vozes da integralidade, fruto da parceria com o Cict/Fiocruz, realizado entre janeiro e março de 2006, dirigido por Sérgio Brito, e a exposição de fotos Olhares da integralidade - produzida em 2006 e lançada no encontro Ensinar saúde, promovido pelo Lappis -, que teve como tema os locais visitados para a realização desse vídeo. O documentário permitiu a volta ao campo de pesquisa um ano após o primeiro contato e o registro, por meio de som e imagem, dos avanços, das dificuldades e das conquistas ocorridos em cada uma das experiências, bem como do impacto que a visita dos pesquisadores havia produzido em cada local. Durante a realização do documentário, percebemos que o registro visual traduzia visões acerca da integralidade e das experiências que compuseram a pesquisa. Organizamos, com o apoio da Fiocruz, uma exposição itinerante, a partir daquela já realizada, que permite compartilhar as imagens vistas e vividas no campo e que 
tem gerado novas possibilidades de comunicação e multiplicação dos resultados obtidos no EnsinaSUS.

\section{Notas}

1 Professora Adjunta do Departamento de Planejamento em Saúde do Instituto de Saúde da Comunidade da Universidade Federal Fluminense (UFF). Coordenadora do LupaSaúde. Pesquisadora associada do Lappis. Mestre e doutora em Saúde Pública pela Escola Nacional de Saúde Pública Sergio Arouca (Ensp), Fiocruz. <lilian@vm.uff.br>

2 Professora assistente da Faculdade de Enfermagem da Universidade Estadual do Rio de Janeiro (UERJ). Pesquisadora do Lappis. Mestre em Enfermagem pela Escola de Enfermagem Anna Nery, da Universidade Federal do Rio de Janeiro (UFRJ). $<$ rmhenriques@uol.com.br>

\section{Referências}

KOIFMAN, Lilian; SAIPPA-OLIVEIRA, Gilson. Produção de conhecimento em saúde. In: PINHEIRO, Roseni; MATTOS, Ruben de Araújo (orgs.). Ensinar Saúde: a integralidade e o SUS nos cursos de graduação na área de saúde. Rio de Janeiro: IMS/Uerj, Cepesc, Abrasco, 2006, p. 111-130.

PINHEIRO, Roseni; CECCIM, Ricardo Burg; MATTOS, Ruben de Araújo. Apresentação. In: PINHEIRO, Roseni; MATTOS, Ruben de Araújo (orgs.). Ensinar Saúde: a integralidade e o SUS nos cursos de graduação na área de saúde. Rio de
Janeiro: IMS/Uerj, Cepesc, Abrasco, 2006, p. 7-12.

PINHEIRO, Roseni et al. Operacionalizando a pesquisa: a trajetória e o fio condutor de construção da pesquisa. In: PINHEIRO, Roseni; MATTOS, Ruben de Araújo (orgs.). Ensinar Saúde: a integralidade e o SUS nos cursos de graduação na área de saúde. Rio de Janeiro: IMS/Uerj, Cepesc, Abrasco, 2006, p. 55-66.

VOZES DA INTEGRALIDADE. 2006. Direção de Sérgio Brito. Cict/Fiocruz, Lappis. 